\title{
Recombination Networks as Genetic Markers in A Human Variation Study of the Old World
}

Marta Meléa , Asif Javed ${ }^{b}$, Laxmi Paridac, Jaume Bertranpetit ${ }^{d}$, Francesc Calafelld, ${ }^{*}$

a Institute of Evolutionary Biology (CSIC-UPF), Barcelona; CRG, Barcelona

b IBM, Yorktown Heights, NY; Genome Institute of Singapore

c IBM, Yorktown Heights, NY

d Institute of Evolutionary Biology (CSIC-UPF), Barcelona francesc.calafell@upf.edu

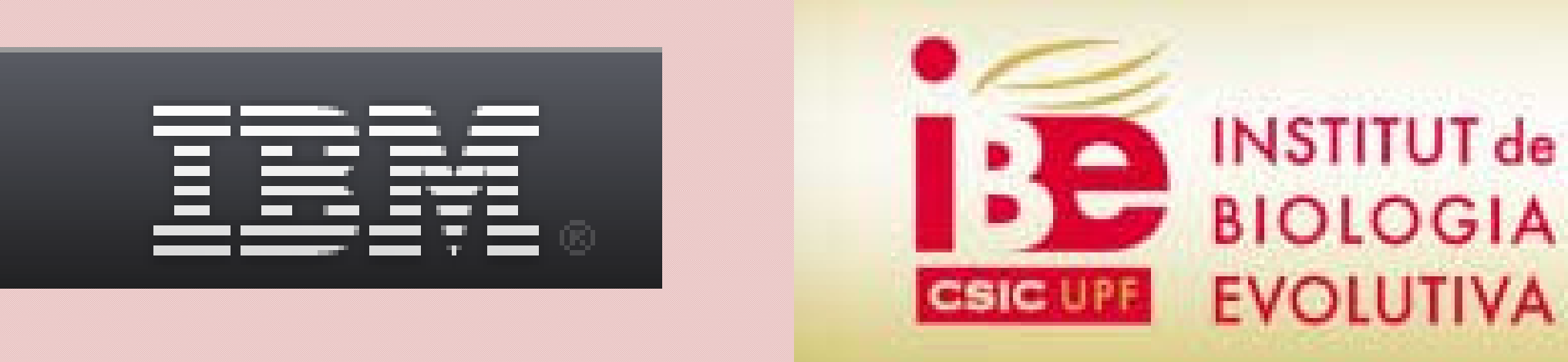

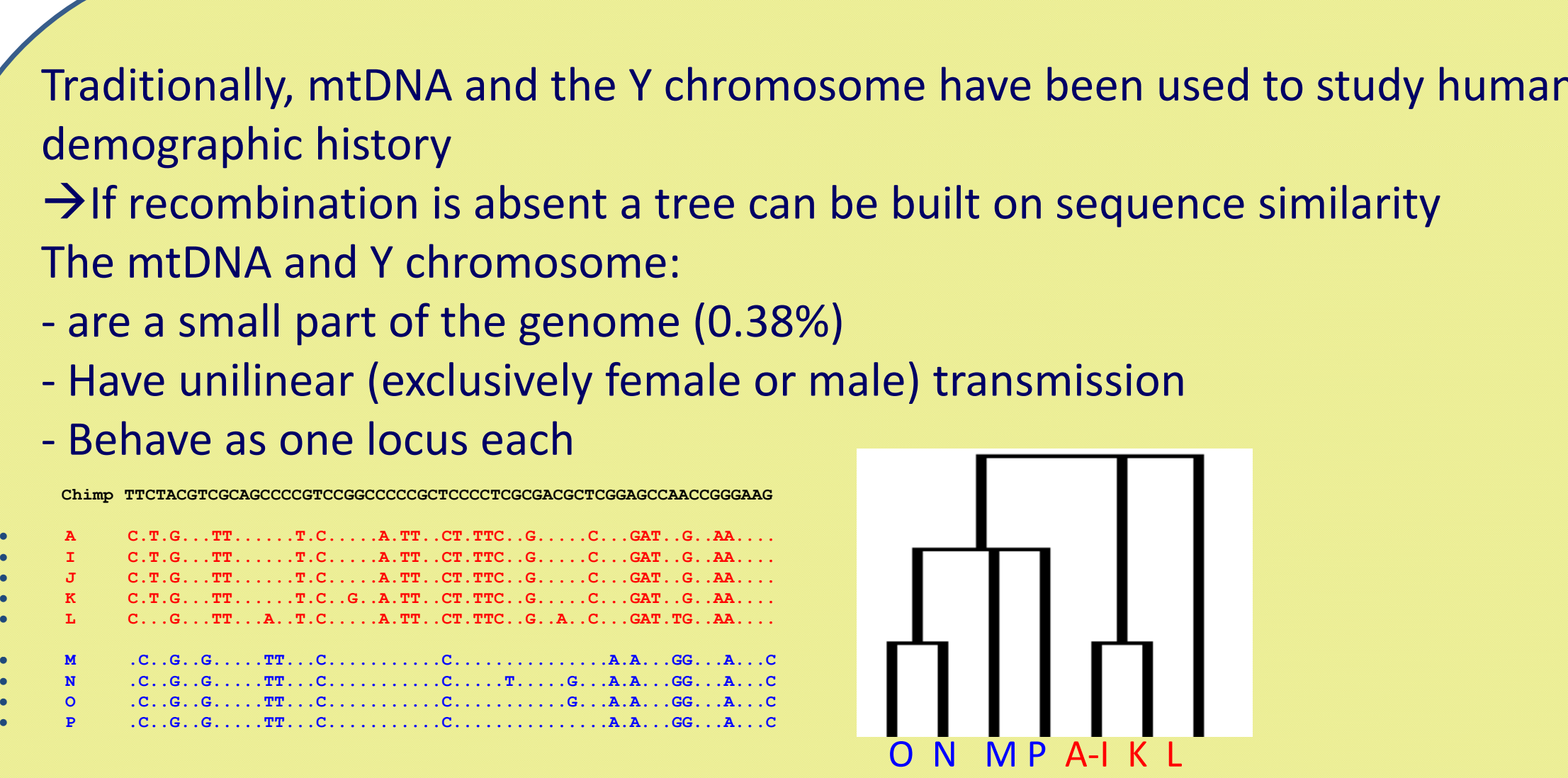

STUDYING HUMAN POPULATION HISTORY BASED ON RECOMBINATION

(Melé et al. Mol Biol Evol. 2012 29:25-30)

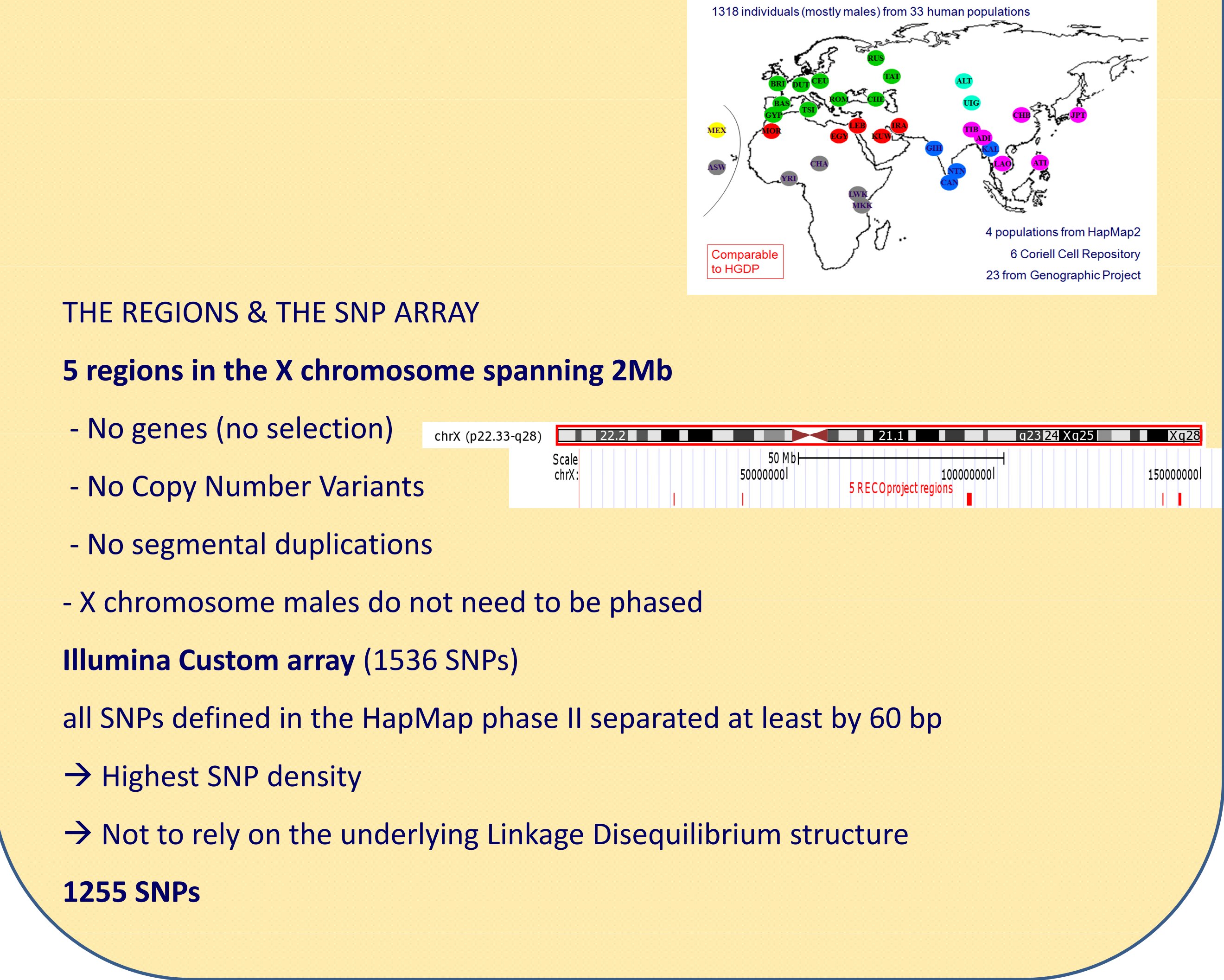

\section{HAPLOTYPES VS SNPS AS GENETIC MARKERS}

(Javed et al. Hum Genet. 2012 131:601-613)

Genetic distances between individuals can be computed as:

1. Fraction of shared SNP alleles

2. Average length of subARG (recombinational) trees linking them

3. Fraction of shared haplotypes (defined as sin Jakobsson et tal. Nature. 2008 451.998-1003)

4. Fraction of fixed-length haplotypes, but, which length?

Which is the optimal length? somewhere between

* High number of different haplotypes (i.e., everyone's different) vs.

* High number of chromosomes in each haplotype (everyone's the same)

Informativeness: Product between average number of haplotypes and average

number of chromosomes per haplotype e.

$S:$ total number of SNPs; $L$ : window length; $h_{i}$ : number of different haplotypes; $N$ :

sample size

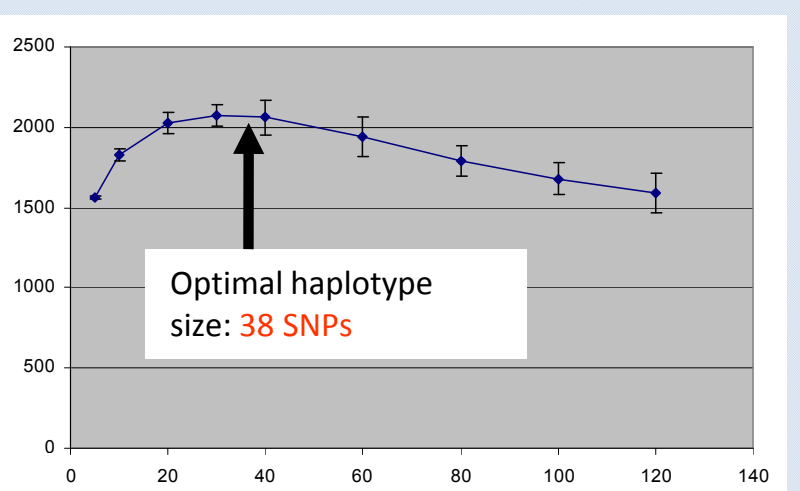

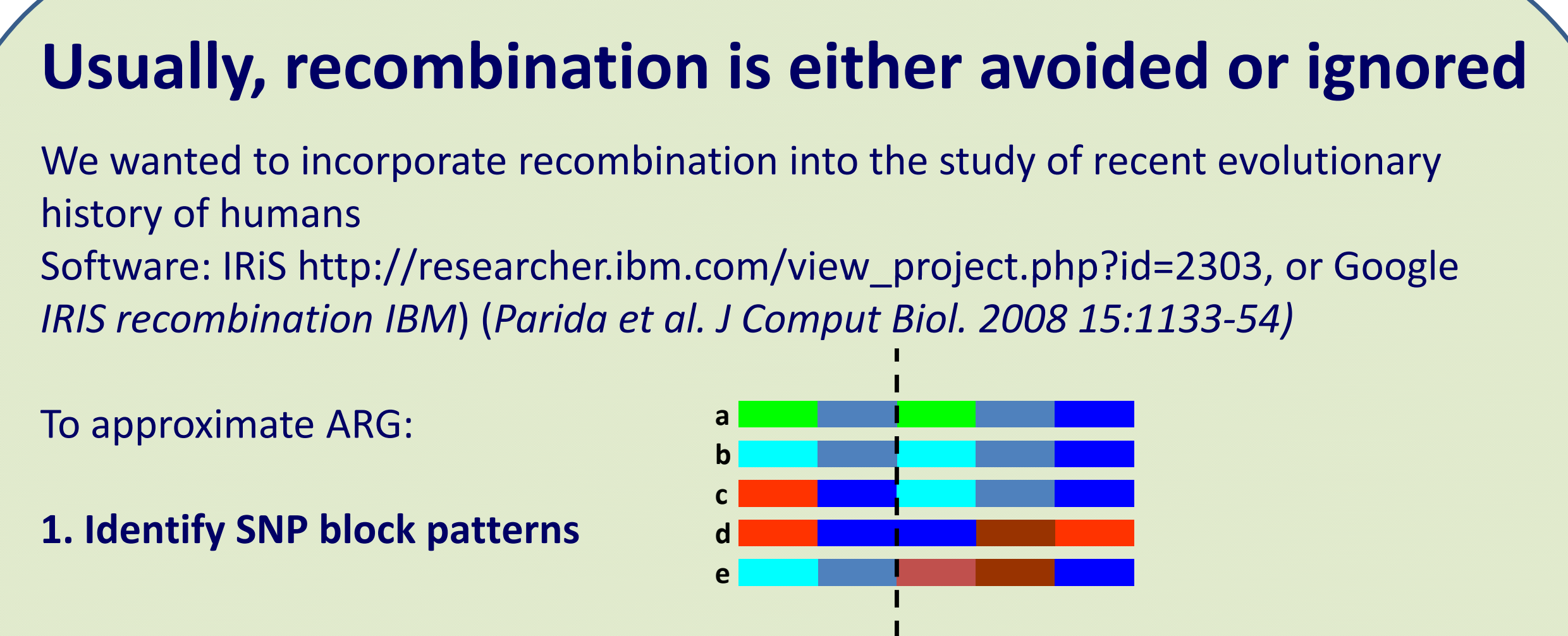

2. Compute segments with no evidence of recombination within

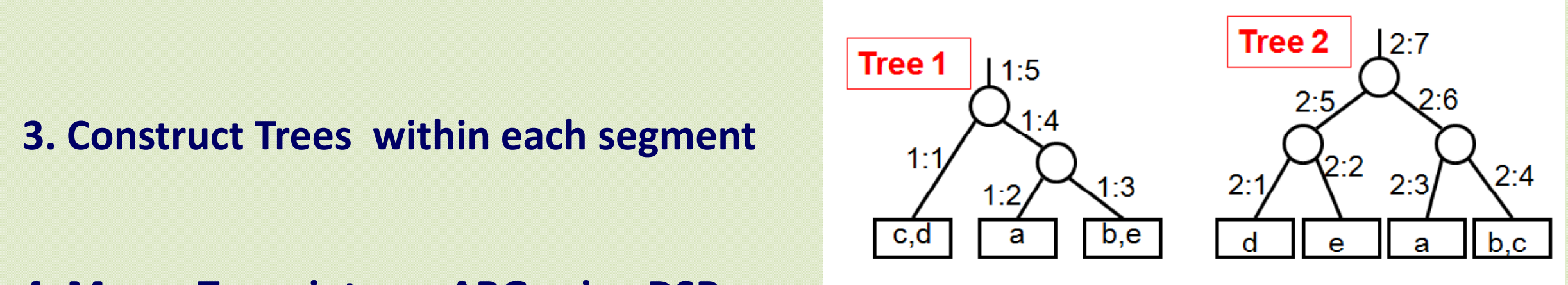

4. Merge Trees into an ARG using DS

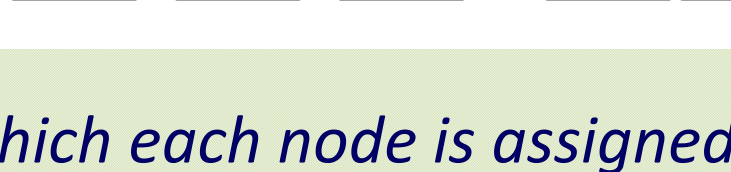

Dominant, Subdominant, or Recombinant.

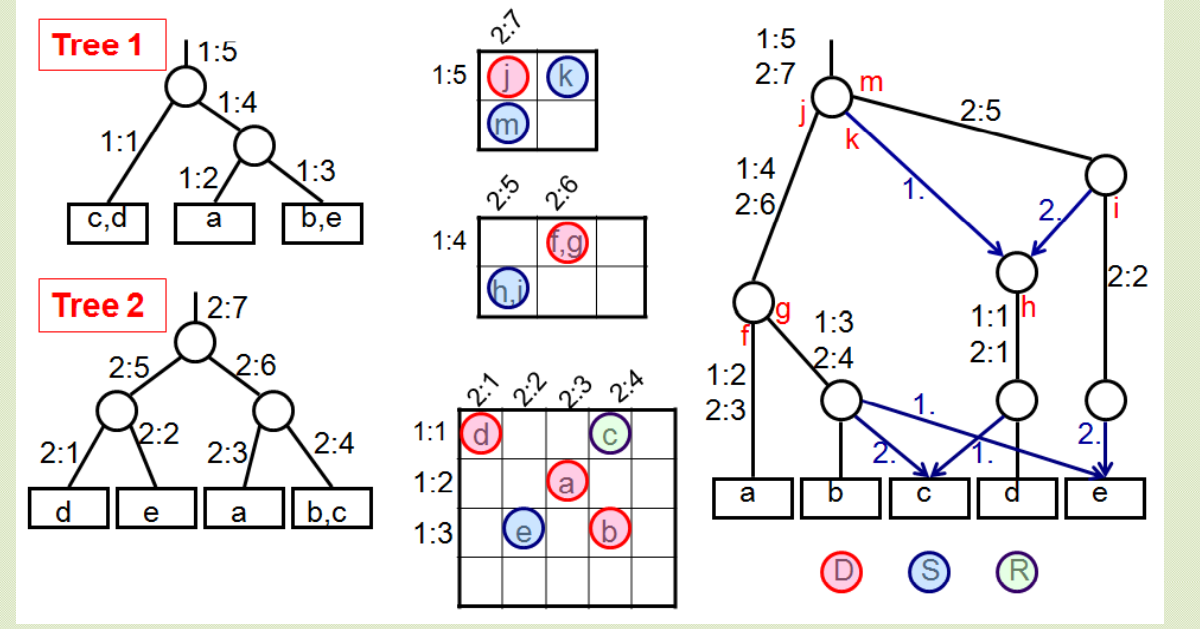

\section{INFERRING THE EFFECTIVE POPULATION SIZE}

Population mutation parameter: $\theta=4 N \mu$

Population mutation parameter: $\theta=4 N_{e} \mu$
Population recombination parameter $\rho=4 N_{e} r\left(=3 N_{e}\right.$ for the X chromosome
$R$

$\begin{aligned} & \rho \text { can be estimated from the number of recombinations (R) } \\ & \dot{a} / \text { la Watterson, after correcting for Ris' sensitivity }\end{aligned} \quad \rho=\frac{R}{n-1}$

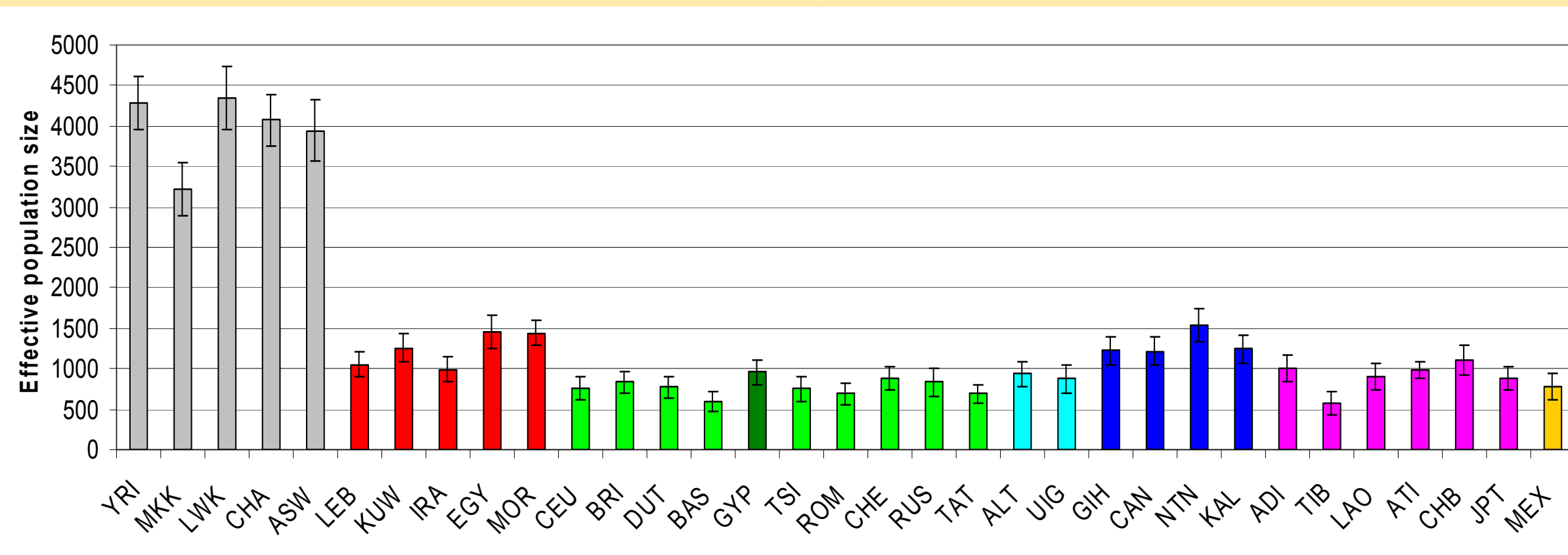

$\mathrm{Ne} \sim 4000$ in Sub-Saharan Africans and 500-1500 elsewhere

Consistent OOA hypothesis, and challenges the received wisdom of $\mathrm{Ne}=10,000$

Unexpected higher effective population size in South Asians

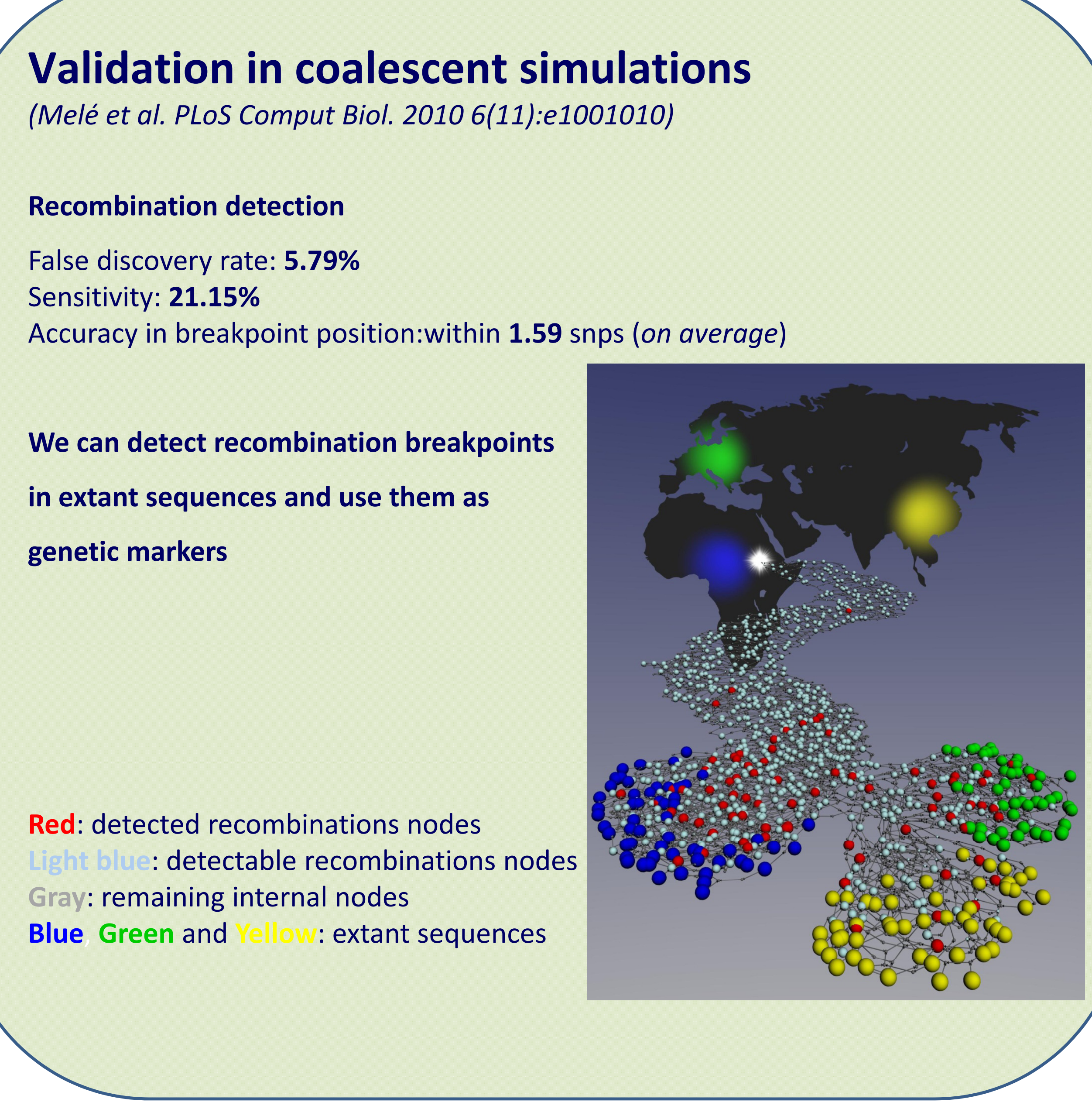

RECOMBINATIONAL DIVERSITY AND MIGRATORY PATHS Recombinational diversity vs distance from East Africa (Southern Arabian vs. Sin routes)

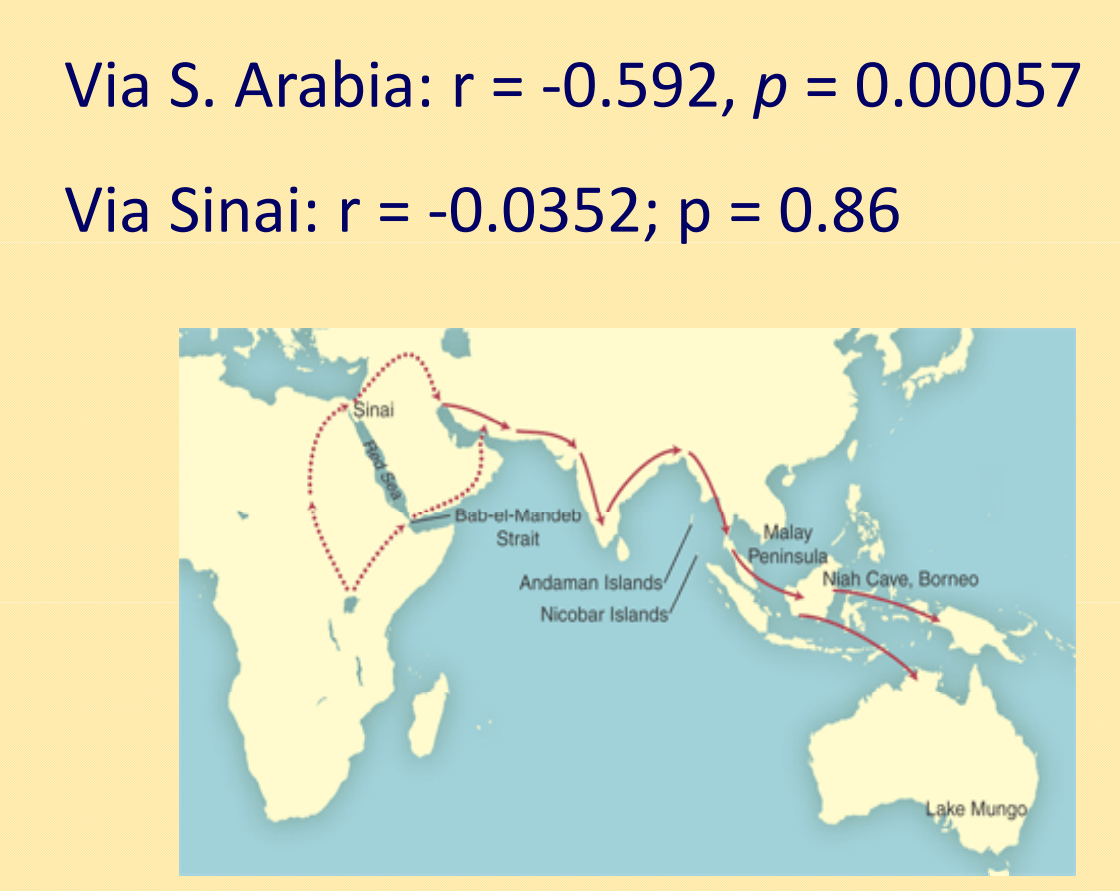

Recombinational diversity is also correlated in non
India: evidence for a mauration phase in India?

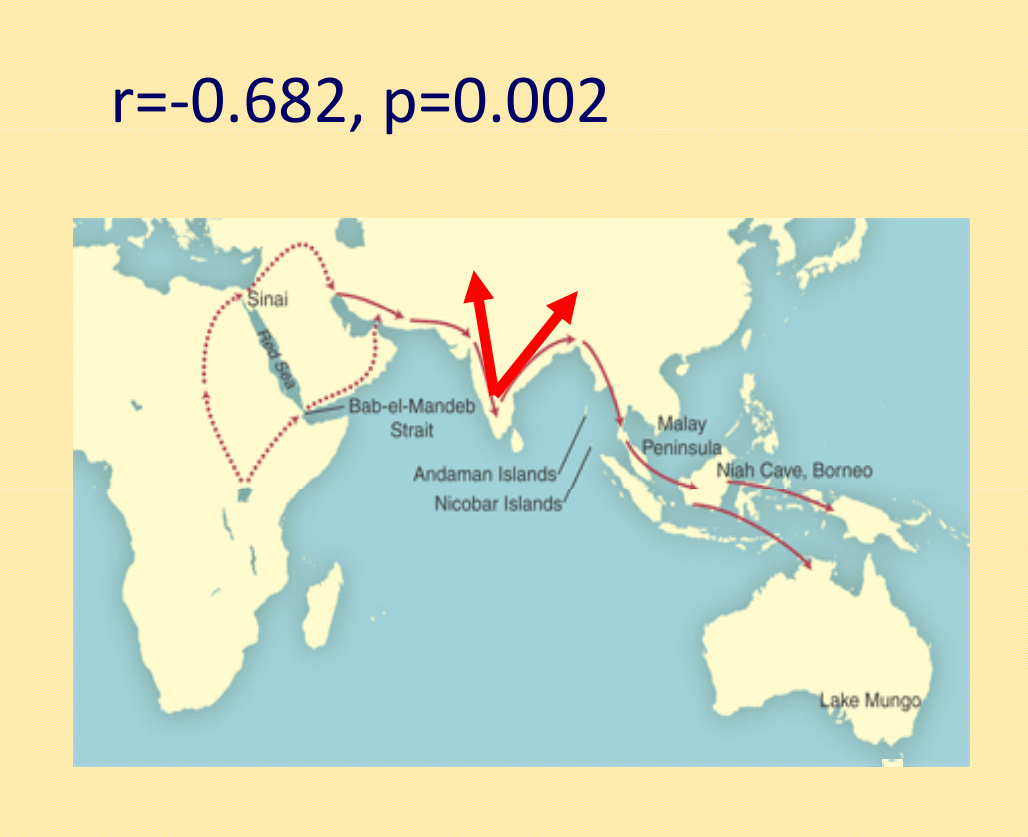

MDS OF PAIRWISE POPULATION DISTANCES

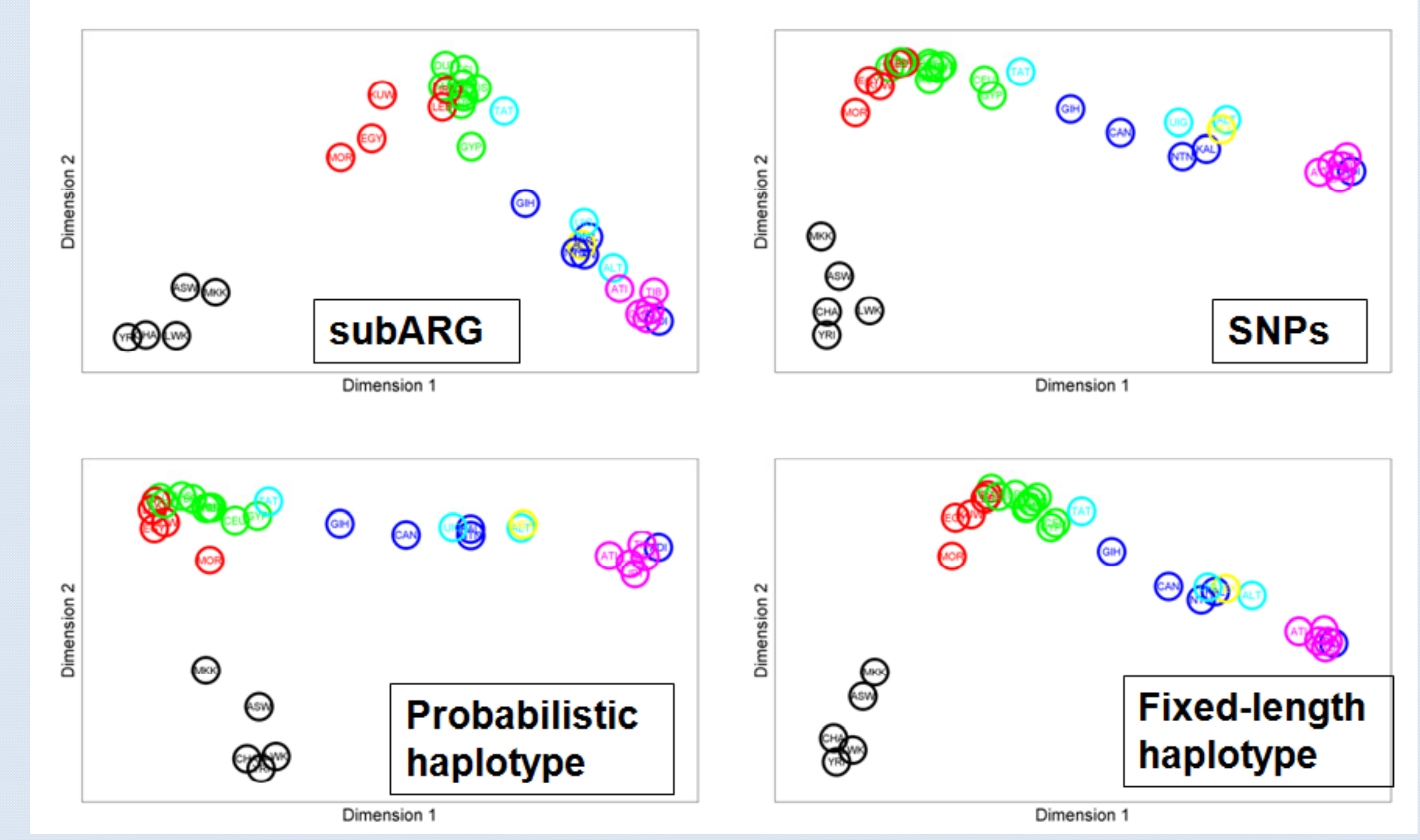
African populations

\section{WHAT HAVE WE LEARNT?}

* We can capture, although with limited sensitivity, ancient recombination events and use them as genetic markers

* Counting accumulated recombination events in a population sample can be used to estimate effective population size. Human Ne values are larger in African populations (duh!), but are higher in South Asians than in other nonAfricans.

* Fixed-length haplotypes seem to yield more information than other ways of analyzing genetic data, porbably because they are the result both of mutation and of recombination.

\begin{tabular}{|ll|}
\hline The Genographic Consortium & Elena Balanovska \\
IBE (CSIC-UPF), Barcelona & Fudan University, Shanghai \\
David COmas & Li lin \\
Begoña Martinez Cruz & Yajun Yang \\
Lebanese American University, & Madurai Kamaraj University, Madurai \\
Beirut & RM Pitchappan \\
Pierre Zalloua & G. Arunkumar \\
Marc Haber & \\
Radboud University Nijmegen & Funding: \\
Medical Center, Nijmegen & IBM \\
Mihai Netea & National Geographic \\
Research Centre for Medical Genetics, & Spanish Ministry of Science and Innovation \\
Moscow \\
Oleg Balanovsky & \\
\hline
\end{tabular}

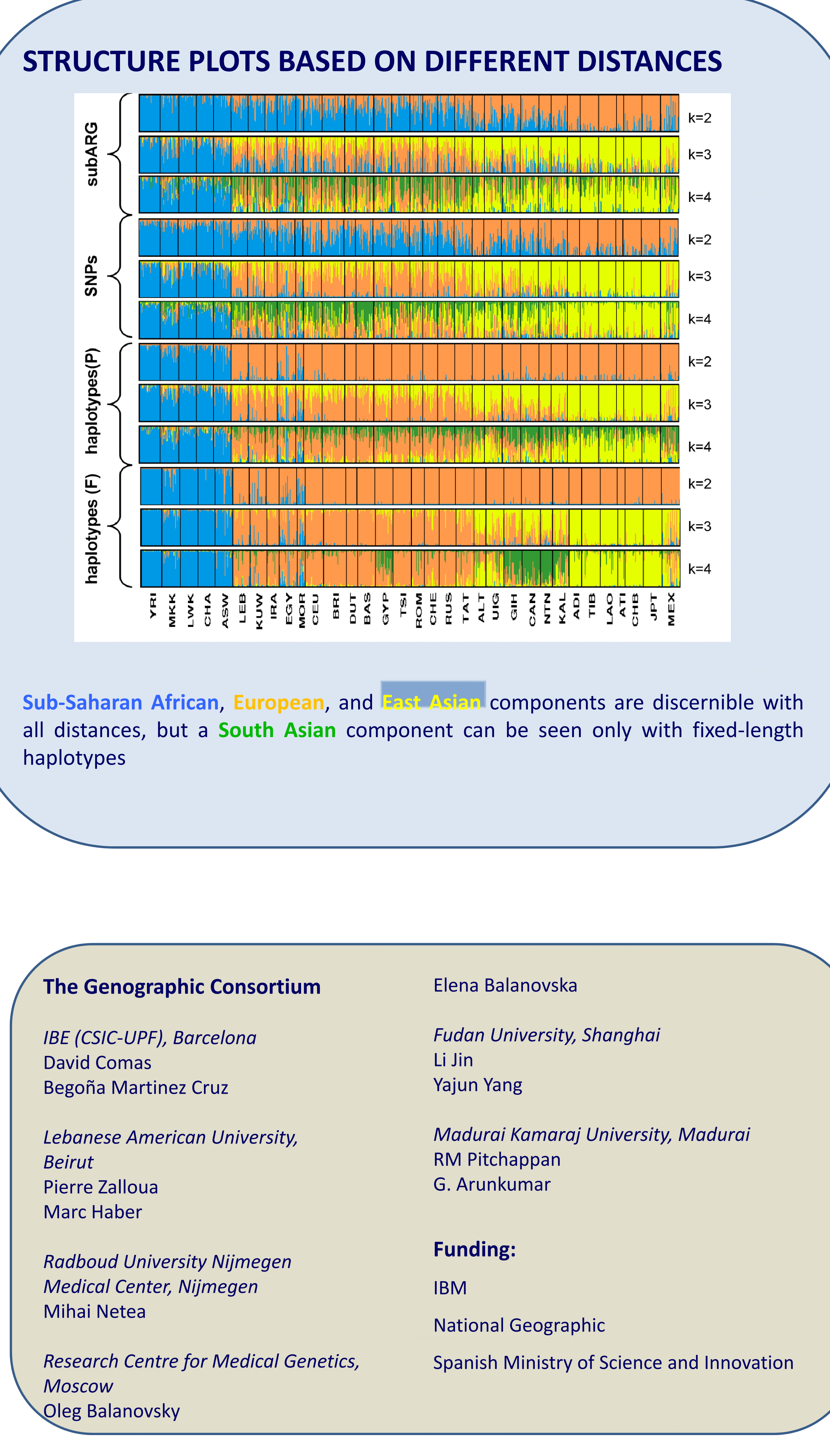

